\title{
Performance of Low-kV Aberration-corrected STEM with Delta-corrector and CFEG in Ultrahigh Vacuum Environment
}

\author{
Takeo Sasaki ${ }^{1}$, Shigeyuki Morishita ${ }^{1}$, Yuji Kohno ${ }^{1}$, Masaki Mukai ${ }^{1}$, Koji Kimoto $^{2}$, and Kazu Suenaga ${ }^{4}$ \\ ${ }^{1 .}$ EM Business unit, JEOL Ltd., Tokyo 196-8558, Japan \\ 2. Research Center for Advanced Measurement and Characterization, National Institute for Materials \\ Science (NIMS), Ibaraki 305-0044, Japan \\ 3. Nanomaterials Research Institute, National Institute of Advanced Industrial Science and Technology \\ (AIST), Ibaraki 305-8565, Japan
}

Single-layered materials, such as graphene or BN, are susceptive to knock-on damage. To reduce this effect, observation of these materials by (scanning) transmission electron microscopy (STEM/TEM) at low acceleration voltage is well-known to be effective. We have developed low-kV STEM/TEM instruments such as TripleC \#1 (CFEG and Delta correctors, 15-60 kV) [1] and TripleC \#2 (Monochromator FEG, and Delta correctors, 15-60 kV) [2] to observe the layered materials with less damage. Even using with lower acceleration voltages from 15 to $60 \mathrm{kV}$, it was found that layered materials were etched with residual gas atoms in a microscope column under electron irradiation. To evaluate the etching of the single-layered materials, a base pressure in a microscope column had better to be changeable from a high vacuum to an ultrahigh vacuum (UHV) range. This paper reports the evacuation system and results of resolution test in the developed UHV low-kV electron microscope.

Figure 1(a) and 1(b) show a scheme and a photo of an evacuation system in TripleC \#3. A CFEG is installed on the top of the microscope. The vacuum of the CFEG is improved by using three sputter-ionpumps (SIPs) and non-evaporable getter pumps. The pressure of CFEG is maintained at the order of $10^{-9}$ $\mathrm{Pa}$ for stable emission of electrons. The column of the microscope is equipped with a TMP-SCR dry system for rough pumping and SIPs $(150 \mathrm{~L} / \mathrm{s}$ for stage, and $75 \mathrm{~L} / \mathrm{s}$ for CM) and Titanium-sublimation pumps (TSP $400 \mathrm{~L} / \mathrm{s}$ ) for high vacuum differential pumping. The CM chamber, the stage chamber, and the objective lens were sealed with metal O-rings to avoid gas transmission from ambient air. The stage and CM chambers are also connected to the SIPs with metal gasket. Rubber O-rings are used for a sideentry goniometer sealing to keep compatibility of specimen holders. The microscope has a higher-order aberration corrector (Delta corrector) to compensate the six-fold astigmatism as well as spherical aberration. To suppress the disturbances caused by the temperature and atmospheric pressure fluctuation, the microscope is covered with an enclosure chassis.

The vacuum of the specimen chamber was controlled from of $10^{-7}$ to $10^{-5} \mathrm{~Pa}$ by switching the evacuation modes: TMP, SIP, and Ti-sublimation pumping modes. The achieved pressures of the stage, which are measured by a Bayard-Alpert gauge, are $1 \times 10^{-7} \mathrm{~Pa}$ (SIP + TSP), $1 \times 10^{-6} \mathrm{~Pa}$ (SIP + TMP), and $1 \times 10^{-5}$ $\mathrm{Pa}$ (TMP). Figure 2 (a) shows a annular-dark-field (ADF) STEM image of Si[110] taken at 60kV with the dwell time of $32 \mu \mathrm{s} /$ pix, convergence semi-angle of $35 \mathrm{mrad}$, and probe current of about $10 \mathrm{pA}$. After compensation of the 6-fold astigmatism and the spherical aberration, we observed clear 136-pm Si-Si dumbbells as shown in Fig. 2(a). Figure 2 (b) is its Fourier transform (FT). A spot of 96 pm is clearly seen. Figure 2 (c) shows an ADF-STEM image of a single layer graphene heated at $773 \mathrm{~K}$ with $256 \mu \mathrm{s} / \mathrm{pix}$ and $45 \mathrm{pA}$. A hexagonal structure of carbon atoms is clearly visible in the image. Its FT (Fig. 2 (d)) shows (107 pm $)^{-1}$ spot. Thus, the mechanical stability of the UHV column and UHV-evacuationsystem is high enough to achieve atomic resolution in STEM imaging. 
References

[1] T. Sasaki et al., J. Electron Microscopy 59 (2010), p. S7.

[2] S. Morishita et al., Phys. Rev. Lett. 117 (2016), p. 153004.

Acknowledgement

This work was supported by the JST under the Research Acceleration Program.
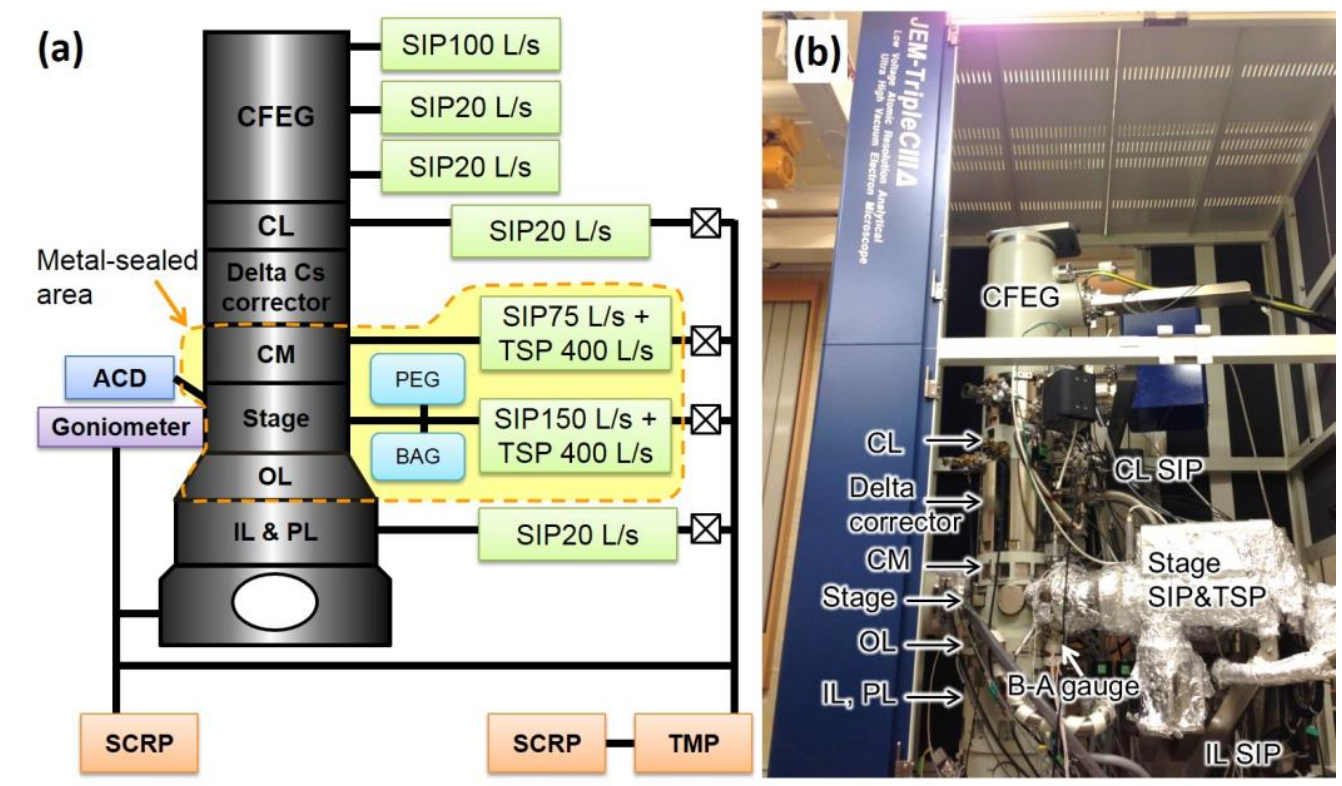

Figure 1. (a) Scheme and (b) Photo of the evacuation system for the low-kV UHV STEM (TripleC \#3).
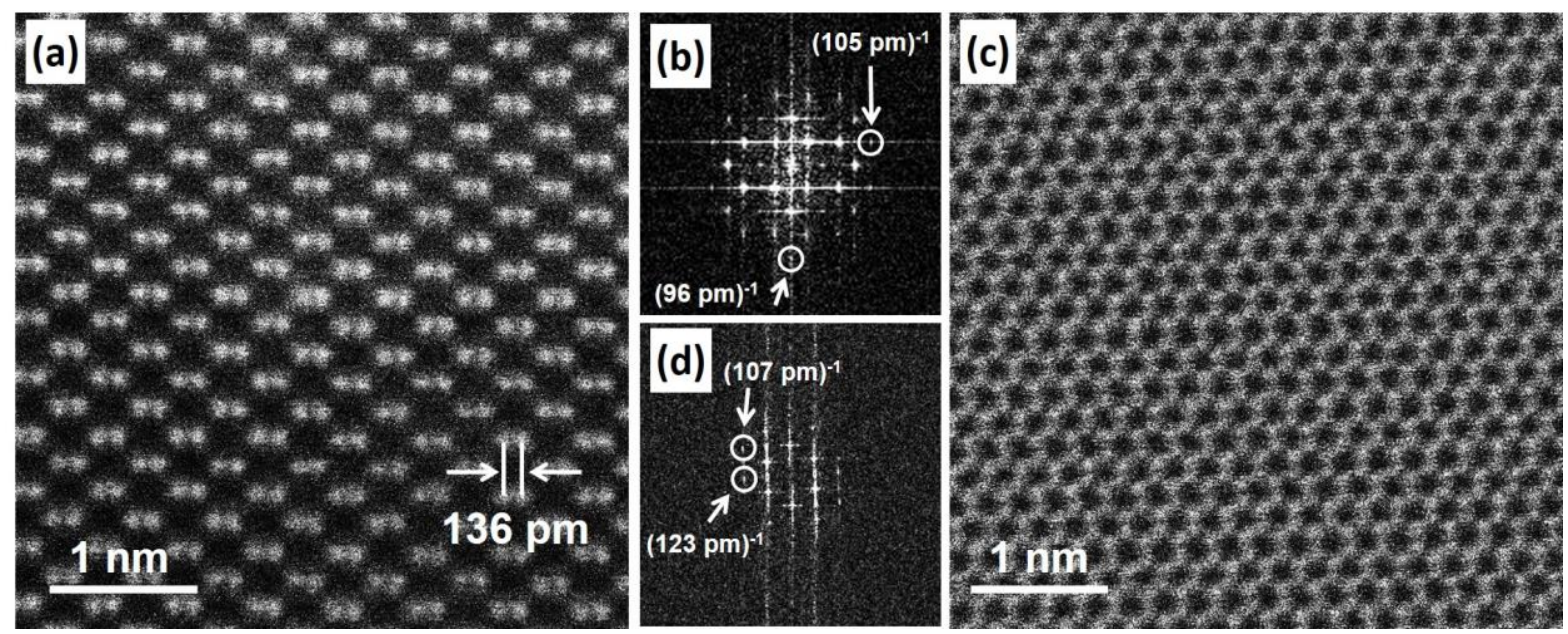

Figure 2. (a) ADF-STEM image of Si[110] crystal taken at $60 \mathrm{kV}$ with convergence semi-angle of 35 $\mathrm{mrad}$, dwell time of $32 \mu \mathrm{s} / \mathrm{pix}$, and the pressure of stage chamber of $4 \times 10^{-7} \mathrm{~Pa}$. (b) Fourier transform (FT) of the image in (a). (c) ADF-STEM image of a heated single layer graphene sheet at $60 \mathrm{kV}$ with convergence semi-angle of $40 \mathrm{mrad}$, dwell time of $256 \mu \mathrm{s} /$ pix, and the pressure of stage chamber of 1.6 $x 10^{-7} \mathrm{~Pa}$. The sample temperature was $773 \mathrm{~K}$. (d) FT of the image in (c). 